\title{
Note on solution regularity of the generalized magnetohydrodynamic equations with partial dissipation
}

\author{
Chuong V. Tran ${ }^{\mathrm{a}}$, Xinwei Yu ${ }^{\mathrm{b}, *}$, Zhichun Zhai ${ }^{\mathrm{b}}$ \\ a School of Mathematics and Statistics, University of St. Andrews, St Andrews, KY16 9SS, United Kingdom \\ ${ }^{\mathrm{b}}$ Department of Mathematical and Statistical Sciences, University of Alberta, Edmonton, AB, T6G 2G1, Canada
}

\section{A R T I C L E I N F O}

\section{Article history:}

Received 13 August 2012

Accepted 19 February 2013

Communicated by Enzo Mitidieri

MSC:

$35 \mathrm{Q} 35$

$76 \mathrm{~B} 03$

76W05

Keywords:

Magnetohydrodynamics

Generalized diffusion

Global regularity

\begin{abstract}
A B S T R A C T
In this brief note we study the $n$-dimensional magnetohydrodynamic equations with hyper-viscosity and zero resistivity. We prove global regularity of solutions when the hyper-viscosity is sufficiently strong.
\end{abstract}

(c) 2013 Elsevier Ltd. All rights reserved.

\section{Introduction}

Consider the $n$-dimensional generalized magnetohydrodynamic ( $n D$ GMHD) equations

$$
\begin{aligned}
& u_{t}+u \cdot \nabla u=-\nabla p+b \cdot \nabla b-v \mathscr{L}_{1}^{2} u, \\
& b_{t}+u \cdot \nabla b=b \cdot \nabla u-\kappa \mathcal{L}_{2}^{2} b, \\
& \nabla \cdot u=\nabla \cdot b=0,
\end{aligned}
$$

where the Laplacians $\Delta$ in the dissipation terms of the momentum and induction equations have been replaced by general negative-definite operators $-\mathscr{L}_{1}^{2}$ and $-\mathscr{L}_{2}^{2}$, respectively. Various forms of these operators have been used in studies concerning the persistence of regularity for classical solutions. In particular, Wu [1] considered $\mathcal{L}_{1}=\Lambda^{\alpha}$ and $\mathcal{L}_{2}=\Lambda^{\beta}$, where $\Lambda:=(-\Delta)^{1 / 2}$, and proved global regularity, that is classical solutions exist for all time, when both $\alpha \geqslant \frac{1}{2}+\frac{n}{4}$ and $\beta \geqslant \frac{1}{2}+\frac{n}{4}$ hold concurrently. This result has been improved by several authors [2-5] (also see [6] for the case of degenerate $\mathcal{L}_{i}$ 's). To date, the best global regularity result for (1)-(3) is the following theorem.

Theorem (Wu 2011 [4]). Consider the GMHD system (1)-(3) with $\mathscr{L}_{1}, \mathfrak{L}_{2}$ defined through Fourier transform as

$$
\widehat{\mathscr{L}_{1} u}(\xi)=m_{1}(\xi) \widehat{u}(\xi), \quad \widehat{\mathscr{L}_{2} b}(\xi)=m_{2}(\xi) \widehat{b}(\xi)
$$

\footnotetext{
* Corresponding author. Tel.: +1 7804925731.

E-mail addresses: chuong@mcs.st-and.ac.uk(C.V. Tran), xinwei2@ualberta.ca, xinweiyu@math.ualberta.ca (X.Yu), zhichun1@ualberta.ca (Z. Zhai).
} 
with

$$
m_{1}(\xi) \geqslant \frac{|\xi|^{\alpha}}{g_{1}(|\xi|)}, \quad m_{2}(\xi) \geqslant \frac{|\xi|^{\beta}}{g_{2}(|\xi|)}
$$

where $g_{1} \geqslant 1$ and $g_{2} \geqslant 1$ are nondecreasing. Assume the initial data belong to $H^{s}$ with $s>1+\frac{n}{2}$. Then the system has a unique global classical solution if the following conditions are satisfied:

$$
\alpha \geqslant \frac{1}{2}+\frac{n}{4}, \quad \beta>0, \quad \alpha+\beta \geqslant 1+\frac{n}{2}, \quad \int_{1}^{\infty} \frac{d s}{s\left(g_{1}(s)^{2}+g_{2}(s)^{2}\right)^{2}}=+\infty .
$$

When $n=2$, conditions much weaker than (6) are sufficient [7]. For example, in the absence of viscosity (i.e. $v=0$ ), global regularity can be secured provided $\beta>2$ (and $g_{2}=1$ ). For $n \geqslant 3$, such a complete removal of $\mathcal{L}_{1}$ is inconceivable. In fact, a drastic weakening of $\mathcal{L}_{1}$ can hardly be expected. The reason is that Eqs. (1)-(3) contain the generalized Navier-Stokes system

$$
u_{t}+u \cdot \nabla u=-\nabla p-\frac{\Lambda^{2 \alpha}}{g_{1}(\Lambda)^{2}} u, \quad \nabla \cdot u=0
$$

as a special case (obtained by setting $b=0$ ), for which the problem of global regularity is still open unless [8]

$$
\alpha \geqslant \frac{1}{2}+\frac{n}{4}, \quad \int_{1}^{\infty} \frac{d s}{s g_{1}(s)^{4}}=+\infty
$$

(also see [9] for the anisotropic case). Hence, before (8), which consists of the first and final conditions (in the absence of $g_{2}$ ) in (6), can be weakened, an improvement concerning the conditions on $\alpha$ and $g_{1}$ in Wu's theorem is highly infeasible.

As indicated by the discussion in the preceding paragraph, the condition on $\alpha$ in (6) is "genuine", and its weakening would be a formidable task. On the other hand, the condition $\beta>0$ appears "technical" and could be removed. The intuitive reason is that for a sufficiently strong $\mathcal{L}_{1}$, bounds can be derived for sufficiently high order derivatives of $u$. Since the induction equation is linear in $b$, this result in turn can be used to prove boundedness for sufficiently high order derivatives of $b$, even in the absence of magnetic diffusion, thereby ensuring regularity. The question is whether the removal of $\beta>0$ can be done without a cost. It turns out that the answer to this question is positive. In fact, we show in this article that (6) can be readily extended to the case $\beta=0$ (more precisely to $\kappa=0$ ). This is accomplished through an application of Lei and Zhou's "weakly nonlinear" energy estimate approach [10], which enables us to derive "almost a priori" bounds for the $H^{1}$ norms of $u$ and $b$. These results are sufficient for obtaining uniform bounds for higher Sobolev norms, hence implying global regularity. To the best of our knowledge, Lei and Zhou first applied this approach to mathematical fluid mechanics in [10].

Now we state our main result.

Theorem 1.1. Consider the following GMHD system

$$
\begin{aligned}
& u_{t}+u \cdot \nabla u=-\nabla p+b \cdot \nabla b-v \mathcal{L}^{2} u, \\
& b_{t}+u \cdot \nabla b=b \cdot \nabla u, \\
& \nabla \cdot u=\nabla \cdot b=0,
\end{aligned}
$$

with

$$
\mathcal{L}:=\frac{\Lambda^{\alpha}}{g(\Lambda)} \text { defined as } \widehat{\mathcal{L} u}(\xi):=\frac{|\xi|^{\alpha}}{g(|\xi|)} \widehat{u}(\xi),
$$

for some function $g(s) \geqslant 1$ defined on $s \geqslant 0$. Let the initial data $u_{0}, b_{0} \in H^{k}$ for some $k>1+\frac{n}{2}$. Then the system has a unique global classical solution if the following conditions are satisfied:

$$
\alpha \geqslant 1+\frac{n}{2}, \quad g(s)^{2} \leqslant C \log (e+s) \quad \text { for some absolute constant } C \text {. }
$$

The following remarks are in order.

- It is clear that (13) extends (6) to the case $\beta=0$.

- $g(s)$ does not need to be nondecreasing.

- In some sense, the condition $g(s)^{2} \leqslant C \log (e+s)$ is weaker than $\int_{1}^{\infty} \frac{d s}{s g(s)^{4}}=+\infty$. For example, given the typical case $g(s) \sim[\log (e+s)]^{\gamma}$, the former requires $\gamma \leqslant 1 / 2$ while the latter requires $\gamma \leqslant 1 / 4$.

The remainder of this article is devoted to the proof of Theorem 1.1. In what follows, we set $v=1$ to simplify the presentation. The adaptation of the proof for other values of $v$ is straightforward. 


\section{Proof of Theorem 1.1}

We present detailed proof for the case $\alpha=1+\frac{n}{2}$, that is $\mathcal{L}:=\frac{\Lambda^{1+n / 2}}{g(\Lambda)}$, or more explicitly

$$
\widehat{\mathscr{L} u}(\xi)=\frac{|\xi|^{1+n / 2}}{g(|\xi|)} \widehat{u}(\xi) .
$$

The case $\alpha>1+\frac{n}{2}$ is much easier to handle and is briefly discussed at the end of this section.

Multiplying (9) and (10) by $u$ and $b$, respectively, and integrating the resulting equations over space, we obtain the standard energy equality

$$
\frac{d}{d t} \frac{\|u\|_{L^{2}}^{2}+\|b\|_{L^{2}}^{2}}{2}+\|\mathcal{L} u\|_{L^{2}}^{2}=0 .
$$

Integrating (15) up to some fixed (but arbitrary) time $T$, we deduce that

$$
u, b \in L^{\infty}\left(0, T ; L^{2}\right), \quad \mathcal{L} u \in L^{2}\left(0, T ; L^{2}\right) .
$$

Note that for any $0 \leqslant \lambda<1+n / 2$ and any $m \geqslant 0$, there is a constant $C$ depending only on $\lambda, m$, and $g$ such that

$$
\|u\|_{H^{m+\lambda}} \leqslant C\left(\|u\|_{L^{2}}+\left\|\mathcal{L} \Lambda^{m} u\right\|_{L^{2}}\right) .
$$

This, together with (16), implies that $u \in L^{2}\left(0, T ; H^{\lambda}\right)$ for any $0 \leqslant \lambda<1+n / 2$.

In the following, we will show that for any $T>0,\|u\|_{H^{k}}$ and $\|b\|_{H^{k}}$ are uniformly bounded over $(0, T)$, or more precisely over $\left(T_{0}, T\right)$ for some $T_{0}$ close enough to $T$. As local well-posedness for (9)-(11) can be proved by standard methods, such uniform bounds secure global regularity. We first show that under the assumption of Theorem 1.1, once (16) holds, the $H^{1}$ norms of $u, b$ have to be much smaller than their $H^{k}$ norms. This makes the trilinear terms in the standard energy method much weaker than its scaling suggests, thereby enabling us to derive $H^{k}$ a priori bounds.

\section{1. $H^{1}$ estimates}

The key to our derivation of estimates in $H^{1}$ is the following lemma, whose proof is given in the Appendix.

Lemma 2.1. Let $g: \mathbb{R}^{+} \mapsto[1,+\infty)$ be such that $g(s)^{2} \leqslant C_{0} \log (e+s)$ for some absolute constant $C_{0}$ and for all $s \geqslant 0$, then there is a constant $C=C(k, n)$ such that

$$
\|\nabla u\|_{L^{\infty}} \leqslant C\left[\|u\|_{L^{2}}+\left\|\frac{\Lambda^{1+\frac{n}{2}}}{g(\Lambda)} u\right\|_{L^{2}} \log \left(e+\|u\|_{H^{k}}\right)\right]
$$

for any $k>1+\frac{n}{2}$.

Remark 2.2. This can be seen as a variant of the classical Brezis-Wainger inequality (see e.g. [11,12]) where $g(\Lambda)=1$ and the $\log$ factor is $\left(\log \left(e+\|u\|_{H^{k}}\right)\right)^{1 / 2}$. It can also be seen as a limiting case of the Sobolev inequalities (see e.g. [13]).

Let $\partial_{i}$ denote a partial derivative. Differentiating (9) and (10) yields

$$
\begin{aligned}
& \left(\partial_{i} u\right)_{t}+u \cdot \nabla \partial_{i} u=-\partial_{i} u \cdot \nabla u-\nabla \partial_{i} p+\partial_{i} b \cdot \nabla b+b \cdot \nabla \partial_{i} b-\mathcal{L}^{2} \partial_{i} u, \\
& \left(\partial_{i} b\right)_{t}+u \cdot \nabla \partial_{i} b=-\partial_{i} u \cdot \nabla b+\partial_{i} b \cdot \nabla u+b \cdot \nabla \partial_{i} u .
\end{aligned}
$$

Multiplying (19) and (20) by $\partial_{i} u$ and $\partial_{i} b$, respectively, integrating the resulting equations in space and summing up over $i$ (noting $\nabla \cdot u=\nabla \cdot b=0$ ) we obtain

$$
\frac{d}{d t} \int_{\mathbb{R}^{n}}\left(\frac{|\nabla u|^{2}+|\nabla b|^{2}}{2}\right) d x+\int_{\mathbb{R}^{n}}|\mathcal{L} \nabla u|^{2} d x \leqslant C\|\nabla u\|_{L^{\infty}} \int_{\mathbb{R}^{n}}\left(|\nabla u|^{2}+|\nabla b|^{2}\right) d x
$$

This implies

$$
\left(\|\nabla u\|_{L^{2}}^{2}+\|\nabla b\|_{L^{2}}^{2}\right)(t) \leqslant\left(\|\nabla u\|_{L^{2}}^{2}+\|\nabla b\|_{L^{2}}^{2}\right)\left(T_{0}\right) \exp \left[C \int_{T_{0}}^{t}\|\nabla u\|_{L^{\infty}}(\tau) d \tau\right] .
$$


Applying Lemma 2.1 we have for any $T_{0}<t$,

$$
\begin{aligned}
\left(\|\nabla u\|_{L^{2}}^{2}+\|\nabla b\|_{L^{2}}^{2}\right)(t) & \leqslant C\left(T_{0}\right) \exp \left[\int_{T_{0}}^{t}\left(\|u\|_{L^{2}}+C\|\mathcal{L} u\|_{L^{2}} \log \left(e+\|u\|_{H^{k}}\right)\right) d s\right] \\
& \leqslant C\left(T_{0}\right) \exp \left[C\left(\int_{T_{0}}^{t}\|\mathcal{L} u\|_{L^{2}} d \tau\right) \log (M(t))\right] \\
& \leqslant C\left(T_{0}\right) M(t)^{C\left(\int_{T_{0}}^{t}\|\mathcal{L} u\|_{L^{2}} d \tau\right)},
\end{aligned}
$$

where

$$
M(t):=\sup _{\tau \in\left(T_{0}, t\right)}\left[e+\|u\|_{H^{k}}+\|b\|_{H^{k}}\right] .
$$

Note that we have used $\|u\|_{L^{2}} \leqslant\left\|u_{0}\right\|_{L^{2}}+\left\|b_{0}\right\|_{L^{2}}$. Also note that the value of $C\left(T_{0}\right)$ changes from line to line.

As $k>1+\frac{n}{2}$, there exists $\lambda$ satisfying

$$
\frac{n}{2} \frac{k}{k-1}<\lambda<1+\frac{n}{2} \text {. }
$$

Now using $\|\mathscr{L} u\|_{L^{2}} \in L^{2}(0, T)$ we see that there exists $T_{0}<T$ such that for all $t \in\left(T_{0}, T\right)$,

$$
C \int_{T_{0}}^{t}\|\mathcal{L} u\|_{L^{2}} d \tau<2 \delta:=\min \left(\frac{(k+\lambda)(k-1)-k\left(k-1+\frac{n}{2}\right)}{k\left(k-1-\frac{n}{2}\right)}, \frac{\lambda-\frac{n}{2}}{k+\lambda}\right) .
$$

Thanks to (25), the right-hand side of (26) is positive since both numbers in the brackets are positive. This allows us to fix $T_{0}$. In what follows, $T_{0}$ is thus fixed.

\section{2. $H^{k}$ estimates}

Let $\partial^{k}$ denote any $k$ th order partial derivative. By applying $\partial^{k}$ to each of (9) and (10), multiplying the resulting equations by $\partial^{k} u$ and $\partial^{k} b$, respectively, and integrating we obtain

$$
\begin{aligned}
\frac{d}{d t}\left(\frac{\left\|\partial^{k} u\right\|_{L^{2}}^{2}+\left\|\partial^{k} b\right\|_{L^{2}}^{2}}{2}\right)+\left\|\mathcal{L} \partial^{k} u\right\|_{L^{2}}= & -\int_{\mathbb{R}^{n}} \partial^{k}(u \cdot \nabla u) \partial^{k} u d x+\int_{\mathbb{R}^{n}} \partial^{k}(b \cdot \nabla b) \partial^{k} u d x \\
& -\int_{\mathbb{R}^{n}} \partial^{k}(u \cdot \nabla b) \partial^{k} b d x+\int_{\mathbb{R}^{n}} \partial^{k}(b \cdot \nabla u) \partial^{k} b d x .
\end{aligned}
$$

Now summing over all $k$ th partial derivatives, and taking advantage of $\nabla \cdot u=\nabla \cdot b=0$, we reach

$$
\frac{d}{d t}\left(\frac{\left\|\nabla^{k} u\right\|_{L^{2}}^{2}+\left\|\nabla^{k} b\right\|_{L^{2}}^{2}}{2}\right)+\left\|\mathcal{L} \nabla^{k} u\right\|_{L^{2}}^{2}=I_{1}+I_{2}+I_{3},
$$

where

$$
\begin{aligned}
& I_{1}=-\sum \int_{\mathbb{R}^{n}}\left[\partial^{k}(u \cdot \nabla u)-u \cdot \nabla \partial^{k} u\right] \partial^{k} u d x, \\
& I_{2}=\sum \int_{\mathbb{R}^{n}}\left[\partial^{k}(b \cdot \nabla b)-b \cdot \nabla \partial^{k} b\right] \partial^{k} u d x+\int_{\mathbb{R}^{n}}\left[\partial^{k}(b \cdot \nabla u)-b \cdot \nabla \partial^{k} u\right] \partial^{k} b d x, \\
& I_{3}=-\sum \int_{\mathbb{R}^{n}}\left[\partial^{k}(u \cdot \nabla b)-u \cdot \nabla \partial^{k} b\right] \partial^{k} b d x .
\end{aligned}
$$

From this we see that

$$
\begin{aligned}
\frac{d}{d t}\left(\frac{\left\|\nabla^{k} u\right\|_{L^{2}}^{2}+\left\|\nabla^{k} b\right\|_{L^{2}}^{2}}{2}\right)+\left\|\mathcal{L} \nabla^{k} u\right\|_{L^{2}}^{2} \leqslant & \sum\left|\int_{\mathbb{R}^{n}} \partial^{l} u \partial^{m} u \partial^{k} u d x\right|+\sum\left|\int_{\mathbb{R}^{n}} \partial^{l} b \partial^{m} b \partial^{k} u d x\right| \\
& +\sum\left|\int_{\mathbb{R}^{n}} \partial^{l} b \partial^{m} u \partial^{k} b d x\right| .
\end{aligned}
$$

The summation is over all possible combinations of partial derivatives satisfying $l+m=k+1, l, m \geqslant 1$. 
- Estimating $\sum\left|\int_{\mathbb{R}^{n}} \partial^{l} u \partial^{m} u \partial^{k} u d x\right|+\sum\left|\int_{\mathbb{R}^{n}} \partial^{l} b \partial^{m} b \partial^{k} u d x\right|$.

These terms can be estimated similarly. So we only present detailed calculations for $\left|\int_{\mathbb{R}^{n}} \partial^{l} b \partial^{m} b \partial^{k} u d x\right|$.

First applying Höder's inequality to the integral yields

$$
\left|\int_{\mathbb{R}^{n}} \partial^{l} b \partial^{m} b \partial^{k} u d x\right| \leqslant\left\|\partial^{l} b\right\|_{L^{2}}\left\|\partial^{m} b\right\|_{L^{2}}\left\|\partial^{k} u\right\|_{\infty} .
$$

Thanks to (25) the following Gagliardo-Nirenberg inequality holds:

$$
\left\|\partial^{k} u\right\|_{L^{\infty}} \leqslant C\|u\|_{L^{2}}^{a}\left\|\Lambda^{k+\lambda} u\right\|_{L^{2}}^{1-a}
$$

with

$$
a=\frac{\lambda-\frac{n}{2}}{k+\lambda} \Longrightarrow 1-a=\frac{k+\frac{n}{2}}{k+\lambda} .
$$

Furthermore, as $l, m \geqslant 1$, we have

with

$$
\left\|\partial^{l} b\right\|_{L^{2}} \leqslant C\|\nabla b\|_{L^{2}}^{\xi}\left\|\nabla^{k} b\right\|_{L^{2}}^{1-\xi} ; \quad\left\|\partial^{m} b\right\|_{L^{2}} \leqslant C\|\nabla b\|_{L^{2}}^{\eta}\left\|\nabla^{k} b\right\|_{L^{2}}^{1-\eta}
$$

$$
\xi=\frac{k-l}{k-1}, \quad \eta=\frac{k-m}{k-1} .
$$

Thus we reach

$$
\left|\int_{\mathbb{R}^{n}} \partial^{l} b \partial^{m} b \partial^{k} u d x\right| \leqslant C\|\nabla b\|_{L^{2}}\left\|\nabla^{k} b\right\|_{L^{2}}\|u\|_{L^{2}}^{a}\left\|\Lambda^{k+\lambda} u\right\|_{L^{2}}^{1-a} .
$$

As $a>0$ we have $1+1-a<2$ and therefore can apply Young's inequality to get

$$
\left|\int_{\mathbb{R}^{n}} \partial^{l} b \partial^{m} b \partial^{k} u d x\right| \leqslant C\|\nabla b\|_{L^{2}}^{\frac{2}{1+a}}\left\|\nabla^{k} b\right\|_{L^{2}}^{\frac{2}{1+a}}\|u\|_{L^{2}}^{\frac{2 a}{1+a}}+\varepsilon\left\|\Lambda^{k+\lambda} u\right\|_{L^{2}}^{2} .
$$

Now using $\|u\|_{L^{2}} \leqslant\left\|u_{0}\right\|_{L^{2}}+\left\|b_{0}\right\|_{L^{2}}$ and (17) we conclude that

$$
\left|\int_{\mathbb{R}^{n}} \partial^{l} b \partial^{m} b \partial^{k} u d x\right| \leqslant C\|\nabla b\|_{L^{2}}^{\frac{2}{1+a}}\left\|\nabla^{k} b\right\|_{L^{2}}^{\frac{2}{1+a}}+\varepsilon\left[\left\|\mathcal{L} \Lambda^{k} u\right\|_{L^{2}}^{2}+1\right]
$$

for $\varepsilon$ as small as necessary.

As the other term can be estimated similarly, we obtain, after taking an appropriate value of $\varepsilon$,

$$
\begin{aligned}
\sum\left[\left|\int_{\mathbb{R}^{n}} \partial^{l} u \partial^{m} u \partial^{k} u d x\right|+\left|\int_{\mathbb{R}^{n}} \partial^{l} b \partial^{m} b \partial^{k} u d x\right|\right] \leqslant & C\left[\|\nabla u\|_{L^{2}}^{\frac{2}{1+a}}\left\|\nabla^{k} u\right\|_{L^{2}}^{\frac{2}{1+a}}+\|\nabla b\|_{L^{2}}^{\frac{2}{1+a}}\left\|\nabla^{k} b\right\|_{L^{2}}^{\frac{2}{1+a}}\right] \\
& +\frac{1}{4}\left[\left\|\mathcal{L} \Lambda^{k} u\right\|_{L^{2}}^{2}+1\right] .
\end{aligned}
$$

Remark 2.3. Note that the $H^{1}$ estimates play crucial roles here. Without them we would have to use

$$
\left\|\partial^{l} b\right\|_{L^{2}} \leqslant\|b\|_{L^{2}}^{\xi}\left\|\nabla^{k} b\right\|_{L^{2}}^{1-\xi}, \quad\left\|\partial^{m} b\right\|_{L^{2}} \leqslant C\|b\|_{L^{2}}^{\eta}\left\|\nabla^{k} b\right\|_{L^{2}}^{1-\eta}
$$

with

$$
1-\xi=\frac{l}{k}, \quad 1-\eta=\frac{m}{k}
$$

and end up with

$$
\left|\int_{\mathbb{R}^{n}} \partial^{l} b \partial^{m} b \partial^{k} u d x\right| \leqslant C\left\|\nabla^{k} b\right\|_{L^{2}}^{\frac{l+m}{k}}\left\|\Lambda^{k+\lambda} u\right\|_{L^{2}}^{1-a} .
$$

Now applying Young's inequality would yield the term $\left\|\mathcal{L} \Lambda^{k} u\right\|^{\gamma}$, where $\gamma>2$, because

$$
\frac{l+m}{k}+1-a=\frac{k+1}{k}+1-a=2+\frac{1}{k}-\frac{\lambda-\frac{n}{2}}{k+\lambda}=2+\frac{k+\lambda-\left(\lambda-\frac{n}{2}\right) k}{k(k+\lambda)}>2
$$

for all $\lambda<1+\frac{n}{2}$. Apparently, such a term is beyond the control of the available dissipation term. 
- Estimating $\sum\left|\int_{\mathbb{R}^{n}} \partial^{l} b \partial^{m} u \partial^{k} b d x\right|$.

We first apply Hölder's inequality

$$
\left|\int_{\mathbb{R}^{n}} \partial^{l} b \partial^{m} u \partial^{k} b d x\right| \leqslant\left\|\partial^{l} b \partial^{m} u\right\|_{L^{2}}\left\|\partial^{k} b\right\|_{L^{2}} .
$$

Now the standard calculus inequality (see e.g. [14]) gives (recall that $l, m \geqslant 1$ ):

$$
\left|\int_{\mathbb{R}^{n}} \partial^{l} b \partial^{m} u \partial^{k} b d x\right| \leqslant C\left[\|\nabla u\|_{L^{\infty}}\left\|\nabla^{k} b\right\|_{L^{2}}^{2}+\|\nabla b\|_{L^{\infty}}\left\|\nabla^{k} u\right\|_{L^{2}}\left\|\nabla^{k} b\right\|_{L^{2}}\right] .
$$

For the first term on the right-hand side, applying Lemma 2.1 yields

$$
\|\nabla u\|_{L^{\infty}}\left\|\nabla^{k} b\right\|_{L^{2}} \leqslant C\left[1+\|\mathcal{L} u\|_{L^{2}} \log \left(e+\|u\|_{H^{k}}+\|b\|_{H^{k}}\right)\right]\left\|\nabla^{k} b\right\|_{L^{2}} .
$$

For the second term, we resort to the following Gagliardo-Nirenberg inequalities. First, we have

$$
\|\nabla b\|_{\infty} \leqslant C\|\nabla b\|_{L^{2}}^{\xi}\left\|\nabla^{k} b\right\|_{L^{2}}^{1-\xi},
$$

where

$$
\xi=\frac{k-1-\frac{n}{2}}{k-1} \Longrightarrow 1-\xi=\frac{n / 2}{k-1} .
$$

Second,

$$
\left\|\nabla^{k} u\right\|_{L^{2}} \leqslant C\left\|\Lambda^{\lambda} u\right\|_{L^{2}}^{\eta}\left\|\Lambda^{k+\lambda} u\right\|_{L^{2}}^{1-\eta},
$$

where

$$
\eta=\frac{\lambda}{k} \Longrightarrow 1-\eta=\frac{k-\lambda}{k} .
$$

Note that $\lambda<1+\frac{n}{2}<k$. It follows that

$$
\|\nabla b\|_{L^{\infty}}\left\|\nabla^{k} u\right\|_{L^{2}}\left\|\nabla^{k} b\right\|_{L^{2}} \leqslant C\|\nabla b\|_{L^{2}}^{\xi}\left\|\nabla^{k} b\right\|_{L^{2}}^{2-\xi}\left\|\Lambda^{\lambda} u\right\|_{L^{2}}^{\eta}\left\|\Lambda^{k+\lambda} u\right\|_{L^{2}}^{1-\eta} .
$$

Obviously $\xi+\eta \leqslant 2$. Furthermore, thanks to (25), we have

$$
\xi+\eta=\frac{k-1-\frac{n}{2}}{k-1}+\frac{\lambda}{k}=1+\frac{\lambda}{k}-\frac{\frac{n}{2}}{k-1}=1+\frac{(k-1) \lambda-\frac{n}{2} k}{k(k-1)}>1 .
$$

Therefore

$$
2-\xi+1-\eta<2, \quad 2-\xi \geqslant \eta .
$$

This enables us to apply Young's inequality to obtain

$$
\|\nabla b\|_{L^{\infty}}\left\|\nabla^{k} u\right\|_{L^{2}}\left\|\nabla^{k} b\right\|_{L^{2}} \leqslant C\|\nabla b\|^{A}\left\|\nabla^{k} b\right\|^{B}\left\|\Lambda^{\lambda} u\right\|^{C}+\varepsilon\left\|\Lambda^{k+\lambda} u\right\|^{2}
$$

with

$$
A=\frac{2 k\left(k-1-\frac{n}{2}\right)}{(k+\lambda)(k-1)}, \quad B=\frac{2 k\left(k-1+\frac{n}{2}\right)}{(k+\lambda)(k-1)}<2, \quad C=\frac{2 \lambda}{k+\lambda}<2 .
$$

Now by (17) and $\|u\|_{L^{2}} \leqslant\left\|u_{0}\right\|_{L^{2}}+\left\|b_{0}\right\|_{L^{2}}$, we have $\left\|\Lambda^{k+\lambda} u\right\|_{L^{2}} \leqslant C\left(\|u\|_{L^{2}}+\left\|\mathcal{L} \Lambda^{k} u\right\|_{L^{2}}\right) \leqslant C\left(1+\left\|\mathcal{L} \Lambda^{k} u\right\|_{L^{2}}\right)$. Similarly, $\left\|\Lambda^{\lambda} u\right\|_{L^{2}} \leqslant C\left(1+\|\mathcal{L} u\|_{L^{2}}\right)$. So finally we reach

$$
\begin{aligned}
\left|\int_{\mathbb{R}^{n}} \partial^{l} b \partial^{m} u \partial^{k} b d x\right| \leqslant & C\left[1+\|\mathcal{L} u\|_{L^{2}} \log \left(e+\|u\|_{H^{k}}+\|b\|_{H^{k}}\right)\right]\left\|\nabla^{k} b\right\|_{L^{2}} \\
& +C\|\nabla b\|^{A}\left\|\nabla^{k} b\right\|^{B}\left(1+\|\mathcal{L} u\|_{L^{2}}\right)+\frac{1}{4}\left(1+\left\|\mathcal{L} \Lambda^{k} u\right\|_{L^{2}}^{2}\right) .
\end{aligned}
$$

In summary, we have obtained

$$
\begin{aligned}
\frac{d}{d t}\left(\frac{\left\|\nabla^{k} u\right\|_{L^{2}}^{2}+\left\|\nabla^{k} b\right\|_{L^{2}}^{2}}{2}\right)+\left\|\mathcal{L} \nabla^{k} u\right\|_{L^{2}}^{2} \leqslant & C\left[\|\nabla u\|_{L^{2}}^{\frac{2}{1+a}}\left\|\nabla^{k} u\right\|_{L^{2}}^{\frac{2}{1+a}}+\|\nabla b\|_{L^{2}}^{\frac{2}{1+a}}\left\|\nabla^{k} b\right\|_{L^{2}}^{\frac{2}{1+a}}\right] \\
& +\frac{1}{4}\left(1+\left\|\mathcal{L} \Lambda^{k} u\right\|_{L^{2}}^{2}\right)
\end{aligned}
$$




$$
\begin{aligned}
& +C\left[1+\|\mathcal{L} u\|_{L^{2}} \log \left(e+\|u\|_{H^{k}}+\|b\|_{H^{k}}\right)\right]\left\|\nabla^{k} b\right\|_{L^{2}}^{2} \\
& +C\|\nabla b\|^{A}\left\|\nabla^{k} b\right\|^{B}\left(1+\|\mathcal{L} u\|_{L^{2}}\right)+\frac{1}{4}\left(1+\left\|\mathcal{L} \Lambda^{k} u\right\|_{L^{2}}^{2}\right) .
\end{aligned}
$$

Here $A, B$ and $a$ are defined in (35) and (57). Recalling the definition of $M(t)$ in (24), we have

$$
\begin{aligned}
\frac{d}{d t}\left(\left\|\nabla^{k} u\right\|_{L^{2}}^{2}+\left\|\nabla^{k} b\right\|_{L^{2}}^{2}\right) \leqslant & C\left(\|\nabla u\|_{L^{2}}^{\frac{2}{1+a}}+\|\nabla b\|_{L^{2}}^{\frac{2}{1+a}}\right) M(t)^{\frac{2}{1+a}} \\
& +C\left[1+\|\mathcal{L} u\|_{L^{2}} \log (M(t))\right] M(t)^{2}+C\|\nabla b\|^{A} M(t)^{B}\left(1+\|\mathcal{L} u\|_{L^{2}}\right) .
\end{aligned}
$$

Here we have used the fact that by definition $M(t)^{2} \geqslant 1$.

Now recalling the earlier result

$$
\|\nabla u\|_{L^{2}}+\|\nabla b\|_{L^{2}} \leqslant M(t)^{\delta}
$$

where $\delta$ is given by (26). Such $\delta$ satisfies $A \delta+B \leqslant 2, \frac{2}{1+a} \delta+\frac{2}{1+a} \leqslant 2$. By denoting $A(t):=1+\|\mathcal{L} u\|_{L^{2}}$ and using the facts that $M(t)>1, \log M(t)>1$, we conclude

$$
\frac{d}{d t}\left(\left\|\nabla^{k} u\right\|_{L^{2}}^{2}+\left\|\nabla^{k} b\right\|_{L^{2}}^{2}\right) \leqslant C A(t) M(t)^{2} \log (M(t)) .
$$

The integration of this equation, together with the energy inequality, gives

$$
M(t) \leqslant C\left(T_{0}\right)\left[1+\int_{T_{0}}^{t} A(\tau) M(\tau) \log (M(\tau)) d \tau\right] .
$$

Standard Gronwall's inequality then gives

$$
M(t) \leqslant C\left(T_{0}\right)^{\exp \left[C\left(T_{0}\right) \int_{T_{0}}^{t} A(\tau) d \tau\right]}
$$

which is uniformly bounded for all $t \in\left(T_{0}, T\right)$ since $\int_{T_{0}}^{T} A(\tau) d \tau<\infty$.

Therefore we have shown that $\|u\|_{H^{k}},\|b\|_{H^{k}}$ are uniformly bounded over $\left(T_{0}, T\right)$, thus completing the proof.

Remark 2.4. The case $\alpha>1+\frac{n}{2}$ can be proved along the same lines, with each step much easier. More specifically, in this case (16) immediately gives $\|\nabla u\|_{L^{\infty}} \in L^{2}(0, T)$, which leads to a priori $H^{1}$ bounds. This allows us to simply take $\delta=0$ in the subsequent steps.

\section{Acknowledgments}

XY and ZZ are supported by a grant from the NSERC and the Startup grant from the Faculty of Science of University of Alberta. The authors would like to thank the anonymous referees for their valuable comments and suggestions.

\section{Appendix. Proof of Lemma 2.1}

The proof involves some basic facts from Littlewood-Paley theory, which we recall here.

Let $\&$ be the Schwartz class of rapidly decreasing functions and $\widehat{f}(\xi)$ denote the Fourier transform of $f(x)$, i.e.

$$
\widehat{f}(\xi):=\frac{1}{(2 \pi)^{n / 2}} \int_{\mathbb{R}^{n}} e^{-i x \cdot \xi} f(x) d x .
$$

Consider $\phi \in \&$ whose frequency is localized:

$$
\operatorname{Supp} \widehat{\phi} \subset\left\{\xi \in \mathbb{R}: \frac{1}{2} \leqslant|\xi| \leqslant 2\right\}
$$

with $\widehat{\phi}(\xi)>0$ if $\frac{1}{2}<|\xi|<2$. Now define $\phi_{j}$ through $\widehat{\phi_{j}}=\widehat{\phi}\left(2^{-j} \xi\right)$. We can multiply $\phi$ by a normalization constant such that the following holds:

$$
\sum_{j \in \mathbb{Z}} \widehat{\phi}_{j}(\xi)=1, \quad \forall \xi \in \mathbb{R}^{n} \backslash\{0\} .
$$


For any $k \in \mathbb{Z}$ we can define operators $S_{k}$ and $\Delta_{k}$ by

$$
\begin{aligned}
& \widehat{S_{k} f}(\xi):=\left[1-\sum_{j \geqslant k+1} \widehat{\phi}_{j}(\xi)\right] \widehat{f}(\xi) \\
& \widehat{\Delta_{k} f}(\xi):=\widehat{\phi}_{k}(\xi) \widehat{f}(\xi) .
\end{aligned}
$$

The most important properties of the operators $S_{k}, \Delta_{k}$ are the following Bernstein inequalities. For any $1 \leqslant p \leqslant q \leqslant \infty$, and $\beta, \beta^{\prime}$ multi-indices with $\beta \geqslant 0$,

$$
\begin{aligned}
& \left\|S_{k} \partial^{\beta} f\right\|_{L^{q}} \leqslant C 2^{|\beta| k} 2^{k n\left(\frac{1}{p}-\frac{1}{q}\right)}\|f\|_{L^{p}} ; \\
& \left\|\Delta_{k} \partial^{\beta^{\prime}} f\right\|_{L^{q}} \leqslant C 2^{\left|\beta^{\prime}\right| k} 2^{k n\left(\frac{1}{p}-\frac{1}{q}\right)}\|f\|_{L^{p}} .
\end{aligned}
$$

Now we are ready to prove Lemma 2.1. The proof is standard and we omit some calculation details.

Proof of Lemma 2.1. We have

$$
\begin{aligned}
\|\nabla u\|_{L^{\infty}} & \leqslant\left\|S_{-1} \nabla u\right\|_{L^{\infty}}+\sum_{j=0}^{N}\left\|\nabla \Delta_{j} u\right\|_{L^{\infty}}+\sum_{j=N+1}^{\infty}\left\|\nabla \Delta_{j} u\right\|_{L^{\infty}} \\
& \leqslant C\left[\|u\|_{L^{2}}+\sum_{j=0}^{N} \frac{2^{j\left(1+\frac{n}{2}\right)}}{g\left(s_{j}\right)}\left\|\Delta_{j} u\right\|_{L^{2}} g\left(s_{j}\right)+\sum_{j=N+1}^{\infty} 2^{j\left(1+\frac{n}{2}-k\right)} 2^{k j}\left\|\Delta_{j} u\right\|_{L^{2}}\right]
\end{aligned}
$$

where $s_{j} \in\left(2^{j-1}, 2^{j+1}\right)$ is chosen such that

$$
g\left(s_{j}\right) \geqslant \frac{1}{2} \sup _{2^{j-1}<s<2^{j+1}} g(s) .
$$

Now we estimate the second term as follows:

$$
\begin{aligned}
\sum_{j=0}^{N} \frac{2^{j\left(1+\frac{n}{2}\right)}}{g\left(s_{j}\right)}\left\|\Delta_{j} u\right\|_{L^{2}} g\left(s_{j}\right) & \leqslant\left[\sum_{j=0}^{N}\left(\frac{2^{j\left(1+\frac{n}{2}\right)}}{g\left(s_{j}\right)}\left\|\Delta_{j} u\right\|_{L^{2}}\right)^{2}\right]^{1 / 2}\left[\sum_{j=0}^{N} g\left(s_{j}\right)^{2}\right]^{1 / 2} \\
& =C\left[\sum_{j=0}^{N}\left\|\frac{2^{j\left(1+\frac{n}{2}\right)}}{g\left(s_{j}\right)} \widehat{\Delta_{j} u}\right\|_{L^{2}}^{2}\right]^{1 / 2}\left[\sum_{j=0}^{N} \log \left(e+s_{j}\right)\right]^{1 / 2} \\
& \leqslant C N\left[\left.\int_{\mathbb{R}^{n}} \frac{|\xi|^{1+n / 2}}{g(|\xi|)} \widehat{u}(\xi)\right|^{2} d \xi\right]^{1 / 2} \\
& =C N\left\|\frac{\Lambda^{1+\frac{n}{2}}}{g(\Lambda)} u\right\|_{L^{2}} .
\end{aligned}
$$

Here we have used the assumption (13), the definition of $s_{j}$ (75), the Plancherel theorem, and the following facts about $\widehat{\phi}_{j}(\xi)$ : 1. $\operatorname{supp}\left(\widehat{\phi}_{j}\right) \subseteq\left\{2^{j-1}<|\xi|<2^{j+1}\right\} ; 2.0 \leqslant \widehat{\phi}_{j}(\xi) \leqslant 1 \Longrightarrow\left|\widehat{\phi}_{j}(\xi)\right|^{2} \leqslant \widehat{\phi}_{j}(\xi) ; 3 . \sum_{j=0}^{N} \widehat{\phi}_{j}(\xi) \leqslant 1$.

For the third term we have

$$
\begin{aligned}
\sum_{j=N+1}^{\infty} 2^{j\left(1+\frac{n}{2}-k\right)} 2^{k j}\left\|\Delta_{j} u\right\|_{L^{2}} & \leqslant\left[\sum_{j=N+1}^{\infty} 2^{2 j\left(1+\frac{n}{2}-k\right)}\right]^{1 / 2}\left[\sum_{j=N+1}^{\infty} 2^{2 k j}\left\|\Delta_{j} u\right\|_{L^{2}}^{2}\right]^{1 / 2} \\
& \leqslant 2^{\left(1+\frac{n}{2}-k\right) N}\|u\|_{H^{k}} .
\end{aligned}
$$

Summarizing, we have

$$
\|\nabla u\|_{L^{\infty}} \leqslant C\left[\|u\|_{L^{2}}+N\left\|\frac{\Lambda^{1+\frac{n}{2}}}{g(\Lambda)} u\right\|_{L^{2}}+2^{\left(1+\frac{n}{2}-k\right) N}\|u\|_{H^{k}}\right] .
$$

Taking $N$ such that $2^{\left(k-1-\frac{n}{2}\right) N} \approx\|u\|_{H^{k}}$ gives the result. 


\section{References}

[1] J. Wu, Generalized MHD equations, J. Differential Equations 195 (2) (2003) 284-312.

[2] G. Wu, Regularity criteria for the 3D generalized MHD equations in terms of vorticity, Nonlinear Anal. 71 (9) (2009) 4251-4258.

[3] J. Wu, Regularity criteria for the generalized MHD equations, Comm. Partial Differential Equations 33 (1-3) (2008) 285-306.

[4] J. Wu, Global regularity for a class of generalized magnetohydrodynamic equations, J. Math. Fluid Mech. 13 (2011) 295-305.

[5] Y. Zhou, Regularity criteria for the generalized viscous MHD equations, Ann. I. H. Poincare-AN 24 (2007) 491-505.

[6] C. Cao, J. Wu, Global regularity for the 2D MHD equations with mixed partial dissipation and magnetic diffusion, Adv. Math. 226 (2)(2011) $1803-1822$.

[7] C.V. Tran, X. Yu, Z. Zhai, On global regularity of 2D generalized magnetohydrodynamic equations, Preprint, Apr. 2012.

[8] T. Tao, Global regularity for a logarithmically supercritical hyperdissipative Navier-Stokes equation, Anal. PDE 2 (2009) 361-366.

[9] T. Zhang, Global regularity for generalized anisotropic Navier-Stokes equations, J. Math. Phys. 51 (2010) 123503.

[10] Z. Lei, Y. Zhou, BKM's criterion and global weak solutions for magnetohydrodynamics with zero viscosity, Discrete Contin. Dyn. Syst. 25 (2) (2009) $575-583$.

[11] H. Brezis, S. Wainger, A note on limiting cases of Sobolev embeddings and convolution inequalities, Comm. Partial Differential Equations 5 (7) (1980) $773-789$.

[12] H. Engler, An alternative proof of the Brezis-Wainger inequality, Comm. Partial Differential Equations 14 (4)(1989) 541-544.

[13] H. Kozono, Y. Taniuchi, Limiting case of the Sobolev inequality in BMO, with application to the Euler equations, Comm. Math. Phys. 214 (2000) 191-200.

[14] A.J. Majda, A.L. Bertozzi, Vorticity and Incompressible Flow, Cambridge University Press, 2002. 